\title{
Computation of fractal dimension of rock pores based on gray CT images
}

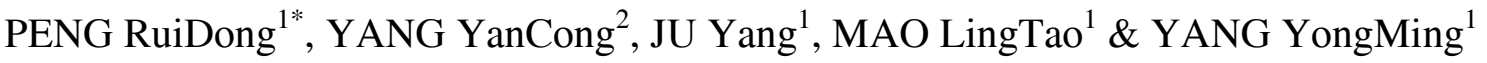 \\ ${ }^{1}$ State Key Laboratory of Coal Resources and Safe Mining, China University of Mining and Technology (Beijing), Beijing 100083, China; \\ ${ }^{2}$ School of Mechanical Electronic and Information Engineering, China University of Mining and Technology (Beijing), Beijing 100083, China
}

Received April 11, 2011; accepted July 20, 2011

\begin{abstract}
The characterization of pore structure in rocks is relevant in determining their various mechanical behaviors. Digital image processing methods integrated with fractal theory were applied to analyze images of rock slices obtained from industry CT, elucidating the characteristics of rock pore structure and the relationship between porosity and fractal dimensions. The gray values of pixels in CT images of rocks provide comprehensive results with respect to the attenuation coefficients of various materials in corresponding rock elements, and these values also reflect the effect of rock porosity at various scales. A segmentation threshold can be determined by inverse analysis based on the pore ratios that are measured experimentally, and subsequently binary images of rock pores can be obtained to study their topological structures. The fractal dimension of rock pore structure increases with an increase in rock pore ratio, and fractal dimensions might differ even if pore ratios are the same. The more complex the structure of a rock, the larger the fractal dimension becomes. The experimental studies have validated that fractal dimension calculated directly from gray CT images of rocks can give an effective complementary parameter to use alongside pore ratios and they can suitably represent the fractal characteristics of rock pores.
\end{abstract}

rock, pore, CT, fractal dimension, image processing

Citation: $\quad$ Peng R D, Yang Y C, Ju Y, et al. Computation of fractal dimension of rock pores based on gray CT images. Chinese Sci Bull, 2011, 56: 3346-3357, doi: $10.1007 / \mathrm{s} 11434-011-4683-9$

As complex geological materials, rocks have discontinuous, non-homogeneous, multi-phase composite structures. Many irregular pores occur at different scales that affect the physical, mechanical and chemical properties of rock materials; such as strength, elastic modulus, permeability, conductivity, wave velocity, particle surface adsorption, and the capacity of a rock reservoir. A significant problem in petroleum exploration, mining, metallurgy, civil and hydraulic engineering is how to understand and quantitatively characterize the evolution of pore structures in rock materials. Thus far, more than 30 kinds of parameters have been proposed to describe rock pore structures [1,2]; these include density or volume fraction of pores (poreratio), pore size and its distribution, and specific surface area. These parameters mainly characterize the average pore structure from a macro-

*Corresponding author (email: prd@cumtb.edu.cn) scopic view. Pore ratio is the most readily available basic parameter, the effect of which is far greater than all the other factors; therefore, studies related to pore ratios are the most common. Determining pore ratios involves use of microscopic image analysis, weighing method, immersion method and mercury, which have been discussed in detail elsewhere $[1,3]$. Because pore structures in rocks are very complex [4-6], advanced measurement techniques have gradually been introduced to describe pore structures at different scales [7]. Pore morphology and microstructure in rocks can be observed and analyzed over different magnifications using optical or scanning electron microscopy. $\mathrm{X}$-ray fluoroscopy methods also have been adopted to observe internal rock structures. A more effective method is Computerized Tomography (CT) by which the density distribution inside materials can be detected. In addition, ultrasound or MRI has been proposed by some researchers. 
The measured results from various experimental testing methods are usually different. However, there should be some relationships among these different results because any measured result is just a particular description of pore structures from a certain perspective.

Since the invention of medical X-ray CT, the inner defects and structures of materials have been detected widely by CT scanning. In the late 1980 s, medical and industrial CT was used to observe the internal structure of rocks. Raynaud et al. [8] obtained CT sliced images of homogeneous gypsum, granite, sandstone, dolomite and other rock samples using a medical $\mathrm{CT}$, and the inner fractures in rocks can be observed clearly from such images. Buyukozturk [9] obtained clear CT images of stones, mortars and vugs in concrete specimens. In the early 1990s, the internal structure of frozen soils was detected by a medical CT, and the mechanism of frozen soil creep was studied [10]. Yang et al. $[11,12]$ were the first to employ this medical CT machine to observe changes in rock microstructure after compression, which constituted the initial application of a real-time CT observation for rocks. In 1999, Ge et al. [13,14] designed a special static loading device integrated with medical CT and successfully solved the problem of real-time scanning by CT during the loading process, which is called dynamic CT testing. Since then, many researchers [15-19] have studied the mesoscopic mechanical behavior of rocks under different loading conditions by means of XCT. The size of CT number and its distribution were studied and the relationship between CT number and rock damage was discussed. Hence steady progress has been made in two areas, specifically the mechanism of evolving defects or cracks and the elucidation of damage constitutive relationships. Ju et al. [20-22] investigated the geometric features and distribution properties of pores in rocks by means of CT scanning tests of sandstone and foam concrete, and then constructed a statistical model of rock pore structure that was used in numerical simulation by finite-element software. The results indicated the important role of CT technology for researching microscopic damage of rock materials. However, some shortcomings of current testing techniques also have gradually been revealed over time. On the one hand, there has been much interest in how to improve the resolution of CT images and imaging speed. Because many fine defects in rock materials need to be detected by high resolution equipment, CT systems with micro- and even nano-focus have been proposed to allow rapid scanning and reconstruction. Furthermore, the algorithm for reconstruction and post-image processing has been improved to reduce artifacts and noise. On the other hand, researchers are currently trying to extract common problems from a large number of experiments to establish controllable damage models to predict damage behavior of rock materials instead of only passively describing the product of damage to rock materials. Thus, an urgent requirement is to strengthen the identification and statistical analysis of CT images so that a pore structure model of rock materials may be built in which several parameters can be controlled and used to reflect the characteristics of the pore structures in rocks.

Fractal theory proposed by Mandelbrot provides a scientific means to describe irregular pore distribution in rocks. Xie first introduced fractal theory into the study of rock mechanics in China [23,24]. Since then many scholars have studied the fractal characteristics of pore structure in rocks. It has been demonstrated by many studies that the pore distribution in rocks is statistically self-similar and fractal dimensions have been introduced to describe the fractal characteristics of pore distribution. Yang et al. [25] produced a fractal model of soil using the weight distribution of particles instead of their size distribution. He et al. [26] calculated the fractal dimension of pores in reservoir rocks according to the expression of capillary pressure and a $\mathrm{J}$ function curve. Li et al. [27] calculated the fractal dimension of cracks during rock failure by counting the box dimension and then evaluated the relationships among fractal dimension, rock composition and stress state. Lian et al. [28] proposed to evaluate rockmass quality by using fractal dimensions to avoid most of the shortcomings of the Rock Quality Designation (RQD). Xue et al. [29] studied multifractal characteristics of pores in soil based on experimental data measured by mercury intrusion methods. Fang et al. [30] calculated the fractal dimension according to different model based on nitrogen adsorption and desorption experiments. Zhou et al. [31] quantified the complexity of the flow boundary by introducing the fractal dimension of boundary shape. Zhang et al. [32] and Tao et al. [33] proposed fractal models of pore volume fraction, particle volume fraction and size distribution of pores or particles based on the models of the Sierpinski gasket and Menger sponge. They validated these models by SEM testing of soils. The calculation of fractal dimension also has been used to analyze CT images of rocks; however, almost all the analyses and calculations are based on the binarization of CT images from gray images to binary images [34-39]. For rocks with simple large pores it is easy to obtain available binary images by approaches such as threshold segmentation or edge detection, and the error caused by this binarization process is negligible. However, for rocks with complex pore structures, some fine pores might be lost to consideration of the subsequent calculations of fractal dimension. If the fractal dimension can be calculated directly according to gray CT images, such errors could be avoided and the calculation process could be simplified. The natural characteristics of gray CT images for rocks and the fractal characteristics of pore structure in rocks are therefore studied in this paper. A method to calculate the fractal dimension of pore structures directly from gray CT images is discussed and validated to better characterize the fractal pore structure. 


\section{Mechanism of CT scanning and test results}

\subsection{Test equipment and samples}

CT is an abbreviation for Computerized Tomography, which is usually actualized by penetrating sections of samples with X-rays and rotating the samples according a certain interval. The intensity of X-rays that have penetrated the sample are measured by the detector and, at the same time, the scanning movements of the X-ray source, the detector and the sample are controlled by the system so that the information required for reconstructing slice images is acquired. Certain algorithms are adopted to analyze these data and thus a series of image slices are reconstructed. Figure 1 shows a typical CT system. Because the entire process, from scanning to reconstructing of images, is controlled by a computer and all the data calculations are made with a computer, such methods are called Computerized Tomography. Based on differences in scanning movement, five generations of CT systems have been developed and scanning velocity has become higher and higher. The 3rd generation of scanning (TR) and the 4th generation of scanning (RO) are commonly employed in present industrial CT systems; the algorithms of parallel-beam reconstruction and fan-beam reconstruction have correspondingly been adopted.

Chinese research related to $\mathrm{CT}$ scanning of rocks has mainly been accomplished at the State Key Laboratory of Frozen Soil Engineering in Lanzhou since the 1990s. The equipment constitutes a medical SOMTOM-plus CT scanner, with spatial resolution of $0.35 \mathrm{~mm} \times 0.35 \mathrm{~mm}$ and a minimum slice height of $1 \mathrm{~mm}$. To improve the scanning precision further, an ACTIS micro focus industrial XCT was introduced at the Geological Scientific Research Institute of Shengli Petroleum Administrative Bureau in Dongying, and a CT225kVFCB micro-CT experimental system was co-designed by Taiyuan University of Technology and Applied Electronics Institute of Academy of Engineering Physics of China, through which pores and cracks on the scale of microns may be distinguished. Figure 2 shows a High Resolution Industry CT Real-time Imaging System (ACTIS300-320/225CT/DR) that was co-designed by the State Key Laboratory of Coal Resources and Safety Mining of China University of Mining \& Technology (Beijing) and American Bio-Imaging Research (BIR) Inc. Using this system, 16-bits images of CT slices could be obtained by quick scanning with resolution at a micron scale. For a sample with the diameter of $25 \mathrm{~mm}$, about $50 \mathrm{~s}$ was required to complete a scan and reconstruct a 16-bit gray image with $1024 \times 1024$ pixels based on 1440 views; both the resolution in the slice plane and the resolution along the slice height reached about $10 \mu \mathrm{m}$. Our tests were conducted using this system.

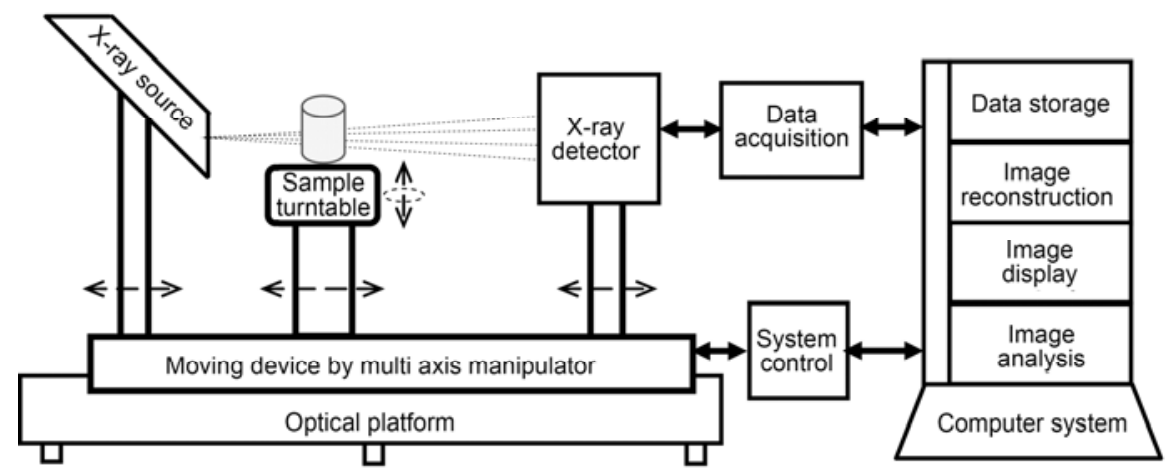

Figure 1 A typical CT system.
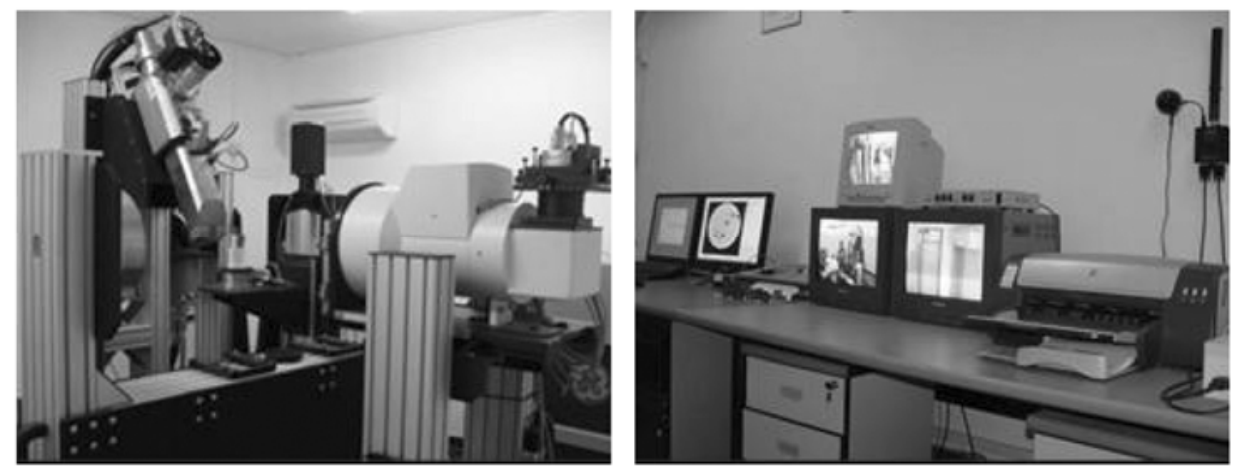

Figure 2 High Resolution Industry CT Real-time Imaging System (ACTIS300-320/225CT/DR). 
For a given CT system, the scanning resolution is strongly related to the sample size. Trying to reduce the sample size as much as possible is helpful for increasing the resolution of the final scanned images. According to features of the CT testing system and the specifications of most rock samples for testing in the laboratory, the rock sample size was determined as a cylinder with a diameter of $25 \mathrm{~mm}$ and height of $50 \mathrm{~mm}$. To compare fractal characteristics of the pore structure among various rock types with different pore ratios, nine kinds of rock samples were considered in the test: basalt, salt rock, coal, mudstone, sandstone, gypsum, oil shale, corundum and concrete. After coring, samples were polished so that their two ends were parallel to one another and vertical to the central axes of the samples. This ensured that a series of slices could be obtained as stable concentric disks when samples were placed on a turntable.

\subsection{Mechanism of CT scanning and imaging}

When X-rays with certain energy pass through samples, because of the photoelectric effect, Compton effect, electron pair effects, Rayleigh scattering, and other complex physical processes, some rays are reflected, scattered or absorbed by samples so that the intensity of X-rays penetrating the samples decreases. The intensity of monoenergetic X-Rays with narrow spectra before and after passing through homogeneous materials agrees with Lambert-Beer's law described as

$$
I=I_{0} \mathrm{e}^{-\mu \Delta x},
$$

where $I_{0}$ is the intensity of incoming X-rays, $I$ is the intensity of penetrated $\mathrm{X}$-rays, $\Delta x$ is the height of samples along the forward direction of $\mathrm{X}$-rays, $\mu$ is the linear attenuation coefficient of materials which changes with variation in energy $E$ of X-rays and the physical properties of the tested materials. When the voltage is less than $200 \mathrm{kV}$, the linear attenuation coefficient depends mainly on Compton scattering and photoelectric absorption, which can be determined by the following equation [40]:

$$
\mu=\rho\left(a+b \frac{Z^{3.8}}{E^{3.2}}\right),
$$

where $\rho$ is the density of material, $Z$ is the atomic number, $a$ is the Kline-Nishina coefficient, $b$ is taken as $9.8 \times 10^{-24}$, and $E$ is the energy of X-ray particles $(\mathrm{keV})$. If the material in the $X Y$ plane is uneven, the attenuation coefficient can be expressed as $\mu(x, y, E, Z, \rho)$, which reflects the combined results of a series of interactions between X-rays and materials. Thus the total attenuation along a path $L$ in a certain direction is

$$
p=\ln \left(I_{0} / I\right)=\int_{L} \mu \mathrm{d} l \approx \sum_{i=1}^{N} \mu \Delta x .
$$

This equation is the projection formula of $\mathrm{X}$-rays in which $p$ is designated as the projection of $\mathrm{X}$-rays after passing through a material. Thereby the integrand function $\mu$ can be solved by a series of projections $p$, and accordingly the distribution of attenuation coefficients in the $X Y$ plane can be obtained. Such a solving process is indeed the principle of image reconstruction utilized by $\mathrm{CT}$. When the material in the $X Y$ plane is divided into $N \times N$ small cells, the attenuation coefficient of each cell can be calculated via the $N^{2}$ times independent measurements. The calculated value of the attenuation coefficient also approximately corresponds to the density of each cell. With medical CTs, a definition of CT number is introduced to describe the attenuation coefficient of human tissue relative to the attenuation coefficient of water. CT number is also known as the Hounsfied number, which is defined as

$$
\frac{\mu-\mu_{w}}{\mu_{w}} \times 1000
$$

where $\mu_{w}$ is the attenuation coefficient of water.

A CT image can be obtained by mapping the attenuation coefficient or the CT number of the material cell to the gray value of each pixel by a certain proportional relation. Because of the effects of quantum statistical laws, partial volume effects, X-ray hardening, multi spectral effects and other factors, certain calibrations are required to ensure that the gray value of pixels in CT images correspond well to the density of the material cell. Because rocks are composed of several types of minerals with various structures, the density of every portion of a rock is different and thus the gray value of CT images of rocks are diverse in different regions. Generally black pixels in CT images indicate objects with lower density, while white indicates high density. The variation of pixel gray from black to white reflects the change of density of the rock cell.

\subsection{CT sliced images of rock samples}

Taking into account the statistical nature of sample slices, only the middle $10 \mathrm{~mm}$ of samples were scanned. The size of scanning view field is $27 \mathrm{~mm}$, and the size of corresponding sliced images is $1024 \times 1024$ pixels. Thus the resolution for each pixel is $26 \mu \mathrm{m}$. Samples were scanned to obtain a slice layer at $1 \mathrm{~mm}$ intervals along the specimen height, and thus 10 sliced images were obtained for each sample. Figure 3 shows one layer of slice images for several kinds of samples. The gray value of each pixel in the CT sliced images reflects the density of the corresponding position. The initial CT slice image is a 16-bit Tiff image and hence has relatively high resolution. However, because of sample density are usually limited to a certain degree and mainly concentrated in low-density regions, the initial image slice represents low brightness and minimal contrast.

A histogram of initial CT sliced images was introduced to analyze the distribution of gray for every pixel. The colors in the four corners of the initial images are directly assigned as black or white when reconstructing images; hence, 


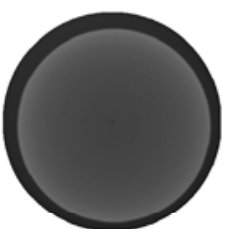

(1) Basalt

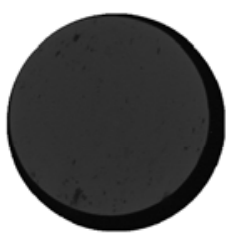

(4) Mud

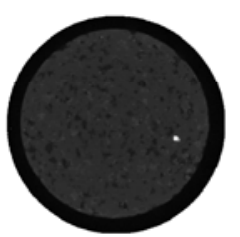

(7) Oil shale

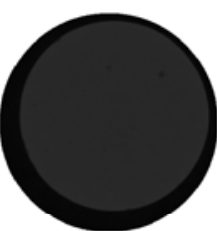

(2) Salt rock

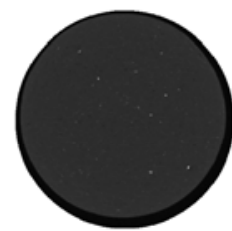

(5) Sandstone

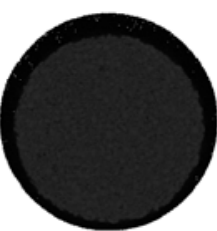

(8) Corundum

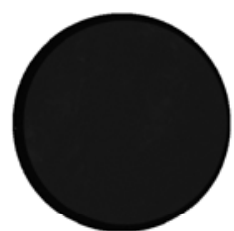

(3) Coal

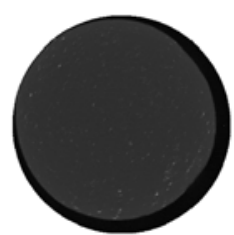

(6) Gypsum

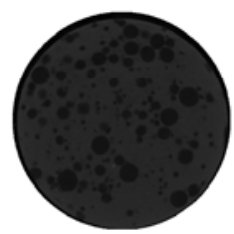

(9) Concrete
Figure 3 Initial CT sliced images of various rock samples.

they are irrelevant to the scanned object and should be omitted when counting gray values. Only the central circular regions of images constitute meaningful data and need to be counted. Furthermore, the edge region of images could be excised to facilitate subsequent image analysis because the statistics of the data slices should be taken into account. Therefore, only the central rectangular regions of rock core samples were statistically analyzed. It is also helpful to eliminate the effect of false images in edges produced by scanning. Figure 4(a) and (c) show the histogram of the initial slice data based on counting the central $512 \times 512$ pixel region of the initial 16-bit Tiff images for coal and concrete samples. It can be seen that the gray value is limited to avery narrow range. Results from other samples also are similar to this case. Because only 8-bit gray images can be directly recognized and displayed in current computer systems, a kind of mapping operation from 16-bit to 8-bit gray level is required to display gray images of CT slices. The usual linear mapping as shown in Figure 5(a) cannot effectively represent the variation in initial gray value. A subsection mapping operation was proposed to enhance image contrast to facilitate observation. Figure 4(b) and (d) show the histogram for 8-bit CT images after conducting subsection gray mapping, and the corresponding CT images are shown in Figure 6. Comparing Figures 3 and 6, it can be seen that the image contrast is effectively improved after subsection gray mapping, and thus the internal pore structure of various rock samples was presented visually.

\section{Analysis of rock pore ratio based on binary images of CT slices}

The density of a rock cell is decreased if there are some pores in the rock cell, such that the color of the pixels that correspond to the rock cell containing pores would be darker than that with no pores. As mentioned previously, the attenuation coefficient of rock cells corresponding to te pixels depends not only on the density and other physical properties of the rock material, but also on a series of interactions between X-rays and rock materials. Especially with an industrial CT scan, both the voltage and current of the X-ray source should be adjusted according to the density and size of the samples. As well, detection results are often not strictly calibrated. Therefore the gray values in CT sliced data can only indicate the differences in density, and the absolute magnitude of the gray values has no strict physical meaning. It is difficult to determine the kind of material that is represented by the pixel simply by comparing
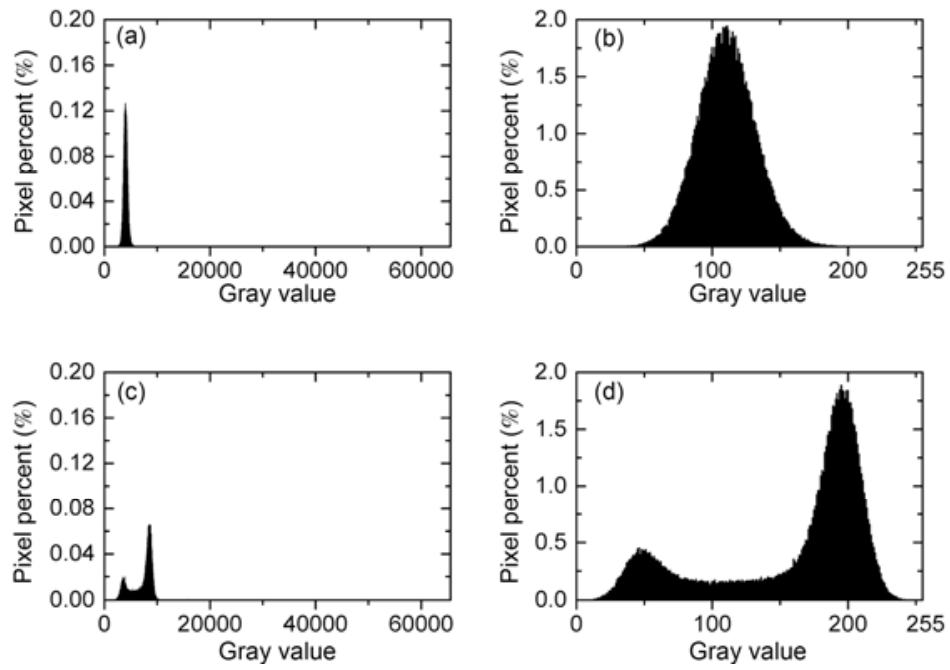

Figure 4 Histograms of initial 16-bit CT slice data and mapped 8-bit CT images. (a) Histogram of initial 16-bits coal slice; (b) histogram of mapped 8-bits coal image; (c) histogram of initial 16-bits concrete slice; (d) histogram of mapped 8-bits concrete image. 

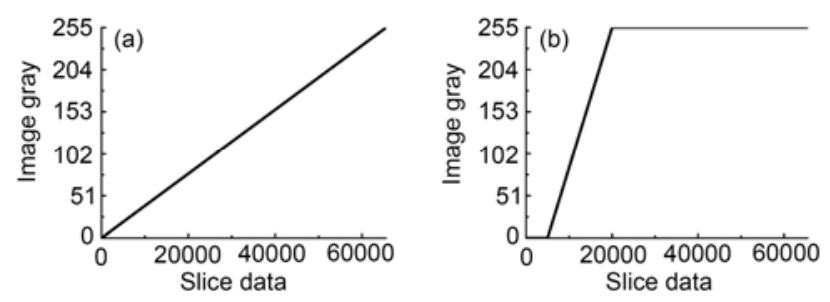

Figure 5 Mapping from 16-bit gray to 8-bit gray.

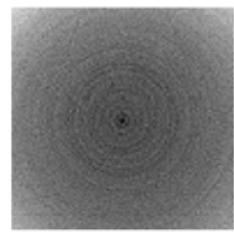

(1) Basalt

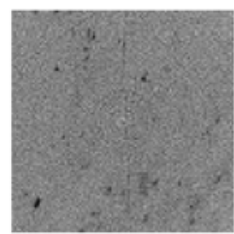

(4) Mud

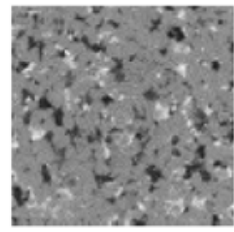

(7) Oil shale

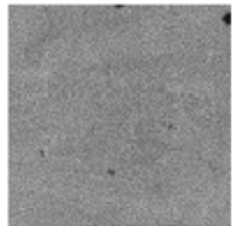

(2) Salt rock

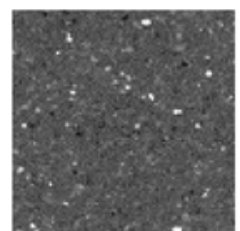

(5) Sandstone

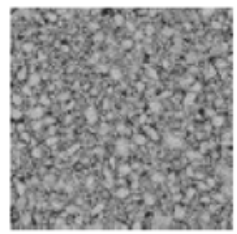

(8) Corundum

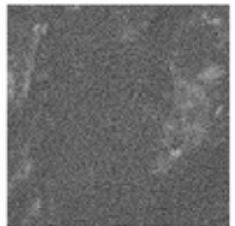

(3) Coal

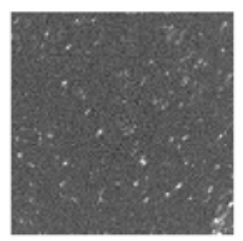

(6) Gypsum

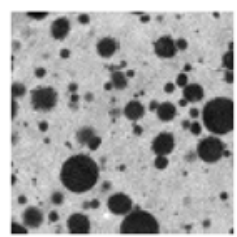

(9) Concrete
Figure 6 CT images of various rock samples after contrast enhancement.

the gray value of each pixel. In addition, when the pixel resolution is too large, the corresponding rock cells might contain various minerals and void. Therefore, the corresponding attenuation coefficient of these rock cells should be the comprehensive reflection of all attenuation coefficients of various substances in the rock cells. It means that the gray value of pixels in CT images is usually the average reflection of the densities of all substances in the corresponding rock cell under certain scanning conditions. To analyze the pore structure of rocks, several types of methods based on image analysis and processing have been utilized, such as threshold segmentation, edge detection, morphological operations (opening, closing, erosion and dilation), and spectral identification. Thus, some binary images were extracted to describe the pore structure.

Based on binary images of rock pore structures, pore ratios can be calculated and the pore topology can be quantitatively characterized. In fact, the advantage of CT scanning is in revealing the inner topological structure of rock pores, such as pore size, pore location distribution and pore connectivity, whereas traditional tests can only determine the value of pore ratios under specific test conditions. It should be more effective for analyzing characteristics of rock pore structures to integrate the CT scanning test and the traditional porosity measuring test. A consideration of the traditional porosity measuring test is also helpful to simplify the complex image processing. The pore ratio is a simple bridge between these two kinds of methods. The value of the pore ratio calculated based on $\mathrm{CT}$ images should be equal or equivalent to that measured from traditional tests.

Among the various approaches to extract binary images of pore structures, threshold segmentation is a simple and effective method. Although the gray value of pores in a CT image is always taken as the smallest one, it is difficult to determine the threshold of the pore gray value because there will usually exist some pores the size of which are less than the pixel resolution, and noise cannot be avoided when scanning and reconstructing. Some CT images of rocks have a histogram similar to Figure 4(c), which indicates the characteristic of two peaks, and thus a particular threshold segmentation algorithm could be introduced for these cases so that better results could be obtained. However, these cases only result where there are many large pores in rocks. Under these conditions, the density of rock cells approximates the density of the rock matrix or the density of pores, and thus its distribution has two peaks. In most cases, the internal pore structure of the rock is very complicated and thus the histogram of CT images of rocks cannot process the characteristics of two peaks, similar to in Figure 4(a). For these cases it is challenging to determine the segmentation threshold directly based on histograms of gray value.

Figure 7 shows pore ratio curves calculated at different thresholds. Correspondingly, the binary images of pore structure obtained by segmentation at the different thresholds are shown in Figure 8. If a low threshold is chosen, the pores cannot be fully extracted and thus the calculated pore ratio is relatively small. If a higher threshold is chosen, some parts of the rock matrix would be extracted and thus the calculated pore ratio is relatively large. The pore ratios measured through traditional permeability tests are: $26 \%$ for mudstone, $27 \%$ for artificial corundum, $28 \%$ for foam concrete. Figure 8 shows that the binary images obtained by segmentation at the threshold corresponding to these pore ratio are satisfactory. Therefore, the segmentation threshold should be determined by inverse analysis based on the pore ratio that is measured experimentally, and then used to segment CT sliced images for obtaining binary images of pore structure as shown in Figure 9. To further improve accuracy, statistics should be used that are appropriate for multi-layer slices; i.e., all the pixels that represent gray values should be counted if they are less than the given threshold in all layers of slices.

\section{Fractal characteristics of rock pores and cal- culation of fractal dimensions}

It has been demonstrated in many studies that pore structure 

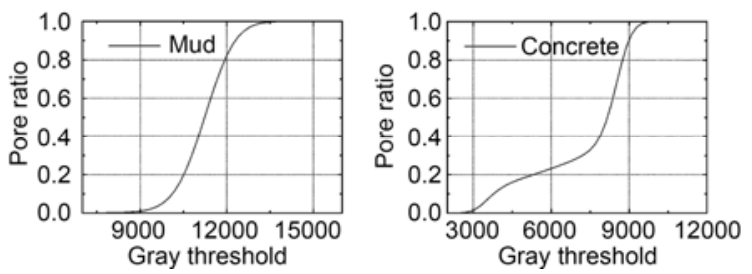

Figure 7 Rock pore ratio under different partition thresholds.

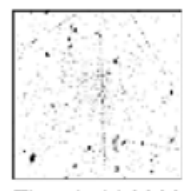

Threshold 9000 Pore ratio $0.98 \%$

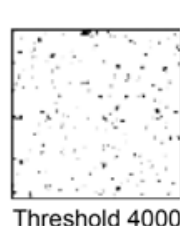
Pore ratio $0.95 \%$

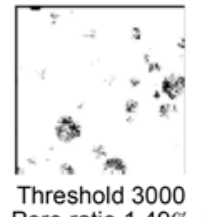
Pore ratio $1.49 \%$

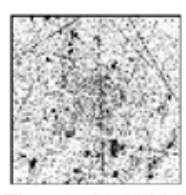

Threshold 10000 Threshold 10700 Threshold 11500 (1) Mud

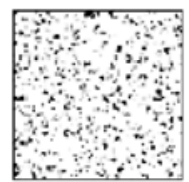

Threshold 5000 Threshold 6400 Threshold 7500 Pore ratio $4.89 \%$ Pore ratio $27.85 \%$ Pore ratio $66.03 \%$

(2) Corundum

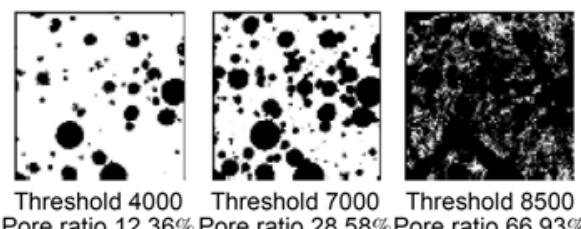

(3) Concrete Pore ratio $8.01 \%$ Pore ratio $26.62 \%$ Pore ratio $62.59 \%$

Figure 8 Binary images of rock pores under different threshold partitions.

in rocks has fractal characteristics and contains a certain self-similarity. When the pores in rocks were observed under different magnifications, both distribution of the relative size of pores and distribution of the relative location of pores was statistically consistent. The calculation of rock pore ratios is strongly related to the range of pore sizes. As more and more micro-pores are considered, the calculated values of the pore ratios also will increase. If only pores with sizes larger than a certain value are considered, the calculated value of the pore ratio will be decreased. Therefore, the characteristics of pore structure in rocks cannot be fully described only using pore ratio and the distribution of pore size. The fractal characteristics of pore structure in rocks should be described by fractal dimensions of pore structure, which can be calculated based on CT images by counting box dimensions. Nowadays the calculation of fractal dimension for digital images is mainly focused on the analysis of binary images, which requires pretreatment of CT images by a particular binarization approach. Although such an approach is helpful to extract the pore structure from CT images, the selection of binarization ap- proaches is likely to affect image analysis and processing, and sometimes might result in losing finely detailed information in some proportion of images. As mentioned previously, the gray value in CT images reflects specific information, and thus different binarization approaches will produce different mesoscopic structures (e.g. holes, cracks), with distinct fractal dimensions. In the following section the methods to calculate the fractal dimensions of images is first discussed, and then binary images and gray images are studied to calculate their fractal dimensions.

\subsection{Calculation of fractal dimensions based on images}

Describing the fractal dimensions of research objects mainly depends on the characteristics of research objects and the aims of the research. Physical fractals in nature usually manifest randomly within some range of scales; i.e., fractal characteristics are shown only in the specific size region from the viewpoint of statistics. Therefore, fractal dimensions are defined as different formulae [24], including Hausdorff dimension $D_{H}$, information dimension $D_{i}$, similar dimension $D_{s}$, correlation dimension $D_{g}$, capacity dimension $D_{c}$, spectral dimension and Lyapunov dimension $D_{l}$. Different definitions can be adapted to calculate fractal dimensions of different research objects. The counting box dimension (CBD) is widely used because its mathematical calculation is relatively simple and it has intuitive physical meaning.

The color mode of images should be considered when we are selecting a specific algorithm to calculate the fractal dimension of images $[41,42]$. The color for a binary CT image has only two kinds of value (usually 0 or 1 ), and thus the calculation of dimensions could be achieved in a two dimensional plane. A gray CT image could be regarded as three dimensional space $\{(x, y, z)\}$, where $x, y$ indicate the position of the pixel in the image plane, and $z$ represents the gray value of the pixel. Hence the gray value of the image produces a rough surface, and thus the calculation of dimensions could be achieved based on tridimensional box covering methods in this three dimensional space.

Binary CT images with the size of $M \times M$ pixels will be considered subsequently in which black pixels with a gray value of 0 indicate pores. The image is divided into a series of grids with an edge length of $\delta_{k}$ and then the number $N_{\delta k}$ of grids containing black pixels is counted. When $\delta_{k} \rightarrow 0$, we can obtain $\lg N_{\delta k} / \lg (1 / \delta) \rightarrow D_{B}$. Thus for a descending sequence $\left\{\delta_{k}\right\}$, the data pair $\left(-\lg \delta_{k}, \lg N_{\delta k}\right)$ can be fitted by the least squares method in logarithmic coordinates. If the correlation coefficient is large enough, the slope becomes just the approximation of the fractal dimension. The decreasing sequence is usually selected by dichotomy method, i.e. $\{M, M / 2, M / 4, M / 8, M / 16, \cdots\}$.

For a gray CT image with a size of an $M \times M$ pixel with gray level 16 (i.e. gray value can vary from 0 to 65535 ), a 
series of box with a bottom edge length of $\delta_{k} \times \delta_{k}$ and a height of $H_{k}$ should be taken to cover the image. The decreasing sequence is usually selected by dichotomy method, and the corresponding box height should be calculated according the gray level as

$$
H_{k}=I_{\max } \cdot \frac{\delta_{k}}{M} .
$$

For a certain box with a different size of $\delta_{k} \times \delta_{k} \times H_{k}$, the number $N_{\delta k}$ of boxes that can cover all the pores in the images is different. Considering the relativity of the gray value in CT images, only the spatial surface corresponding to the gray of all pixels should be covered by the boxes, so

$$
N_{\delta_{k}}=\sum_{n=1}^{2^{2 k}}\left(\frac{\max (I(i, j))-\min (I(i, j))}{H_{k}}\right),
$$

where $I(i, j)$ indicate the set of gray value of all pixels in the $\mathrm{n}^{\text {th }}$ grid corresponding to the size of $\delta_{k}$. The data pair $\left(-\lg \delta_{k}\right.$, $\left.\lg N_{\delta k}\right)$ in the non-scale region can be linearly fitted by the least squares method in logarithmic coordinates and thus a linear equation can be determined as

$$
\lg N_{\delta_{k}}=a \cdot\left(-\lg \delta_{k}\right)+b,
$$

in which slope $a$ is the fractal dimension $D_{C B}$ of pore images.

\subsection{Fractal dimension of rock pores based on binary images of CT slices}

The fractal dimension calculated from binary images of CT slices is shown in Figure 9. Results shown in Figure 9 indicate that the correlation coefficients are generally larger than 0.99; therefore, the calculated fractal dimension is valid. The pore ratio of basalt samples is close to 0 , and thus the corresponding binary image is a pure white image with fractal dimension of 0 and is not listed in Figure 9.

The pore ratios of salt rock samples and coal samples are lower, less than $1 \%$, and the calculated fractal dimension is less than 1. This indicates that the pores in the salt rock and coal contain micro-holes isolated for each other.

The pore ratio of sandstone and oil shale samples are almost equal, at about $15 \%$. However, their fractal dimensions are distinct. The fractal dimension of sandstone samples is 1.75 , while the fractal dimension of oil shale samples is 1.66. Therefore, the pore structure in sandstone is more complex than that in oil shale, as shown in Figures 6 and 9. There are many large pores and a few small pores in oil shale, while there are a few large pores and many fine pores in sandstone.

The pore ratios of mudstone, gypsum, corundum and foam concrete samples are almost equal, at about $27 \%$, but their fractal dimensions are different. The fractal dimension of mudstone and gypsum samples is 1.85 , corundum samples 1.81, and foam concrete samples 1.76. Images in Figures 6 and 9 show that the pores in mudstone and gypsum are more complex than those in corundum, which in turn are more complex than pores in foam concrete.

The fractal dimension of pore images can be well described by calculating binary images of CT slices, but the results of the calculations depend strongly on the segmentation threshold of binarization approaches. As shown in Figure 8 , the binary images obtained according to different segmentation thresholds are dissimilar, and therefore both the pore ratio and the fractal dimension calculated from the binary images are distinct, as listed in Table 1.

The results show that the fractal dimension of pore structures would increase with increasing pore ratio, and furthermore the fractal dimension of pore structures might be distinct even if the pore ratio is equal. The more complex the pore structure, the greater its fractal dimension. Therefore, pore structure can be well characterized by fractal dimension. Especially for pore structures with the same pore ratio, the fractal dimension could effectively characterize their differing complexity.

\subsection{Fractal dimension of rock pores based on gray im- ages of CT slices}

To eliminate the effect of binarization approaches on the calculation of fractal dimensions, gray CT images were directly utilized to calculate fractal dimensions, as shown in Figure 10. It is evident from the results shown in Figure 10 that the correlation coefficients are larger than 0.99 , thus the calculated fractal dimension is valid.

Results of calculations directly from gray images show that the fractal dimension of basalt, rock salt and coal samples are relatively larger, which is different from the results of calculation from binary images. This is caused by the fact that many pixels cannot be extracted in binarization approaches and only a few pores with relatively large sizes can be considered. Such a process is available only when pores smaller than micron scale can be neglected. In fact, rocks usually contain a large numbers of micro-pores smaller than micron scale, which cannot easily be measured by traditional test methods. Therefore the pore ratios measured in the present test were very low and only a few large pores were extracted by binarization approaches based on inverse analysis. However, the density of the rock cells containing these pores would represent infinitesimal changes, which can only be detected by CT and reflected in gray

Table 1 Porosity and fractal dimension of rock pores under different segmentation thresholds

\begin{tabular}{cccccccc}
\hline \multicolumn{2}{c}{ Mud } & & \multicolumn{2}{c}{ Corundum } & & \multicolumn{2}{c}{ Concrete } \\
\cline { 1 - 3 } \cline { 7 - 8 } $\begin{array}{c}\text { Pore ratio } \\
(\%)\end{array}$ & $\begin{array}{c}\text { Fractal } \\
\text { dimension }\end{array}$ & $\begin{array}{c}\text { Pore ratio } \\
(\%)\end{array}$ & $\begin{array}{c}\text { Fractal } \\
\text { dimension }\end{array}$ & $\begin{array}{c}\text { Pore ratio } \\
(\%)\end{array}$ & $\begin{array}{c}\text { Fractal } \\
\text { dimension }\end{array}$ \\
\hline 0.98 & 1.350 & & 0.95 & 1.167 & & 1.49 & 1.287 \\
8.01 & 1.775 & & 4.89 & 1.489 & & 12.36 & 1.602 \\
26.62 & 1.847 & & 27.85 & 1.809 & & 28.58 & 1.762 \\
62.59 & 1.954 & 66.03 & 1.944 & & 66.93 & 1.953 \\
\hline
\end{tabular}




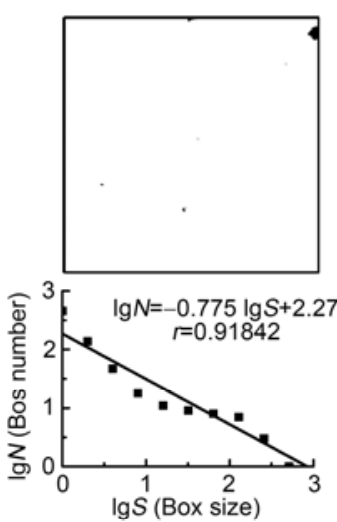

Threshold 7000 Pore ratio $0.17 \%$ Fractal dimension 0.775

(1) Salt rock
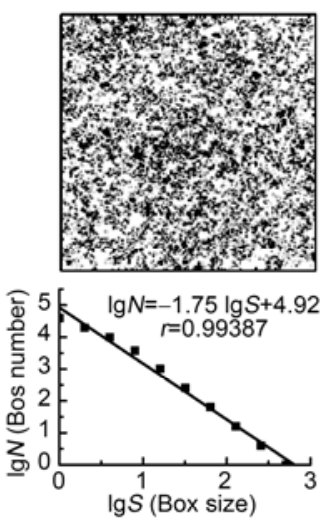
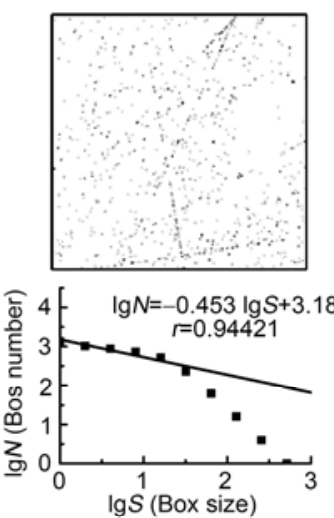

Threshold 3100 Pore ratio 0.51 Fractal dimension 0.453

(2) Coal
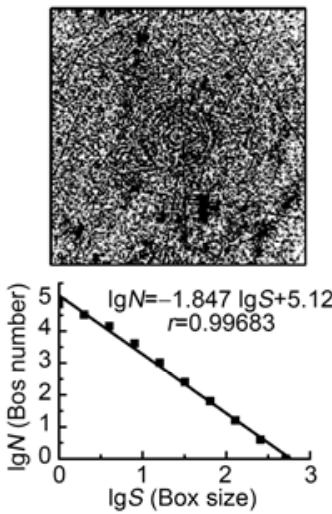
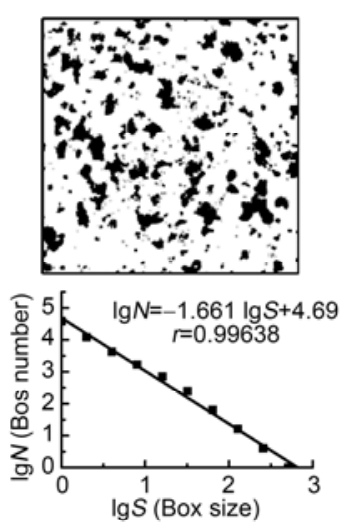

$\lg S$ (Box size)
Threshold 10000 Pore ratio 15.11 Fractal dimension 1.753

(4) Sandstone
Fractal dimension 1.847

(5) Mud eshold 8500 Pore ratio $14.71 \%$

(3) Oil shale
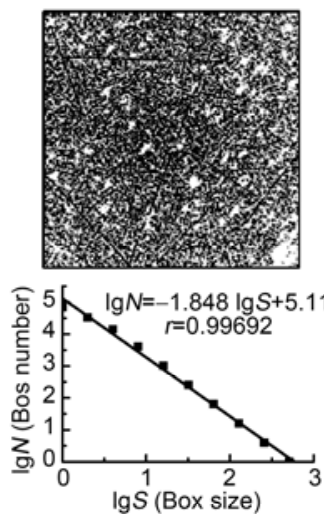
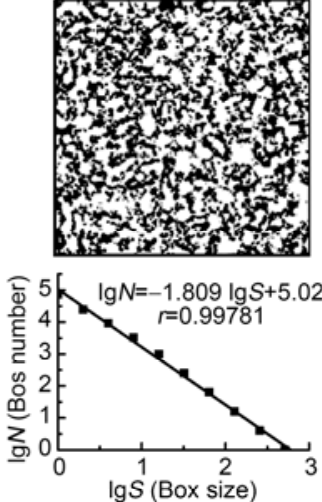

Threshold 6400 Pore ratio $27.85^{\circ}$ Fractal dimension 1.809

(7) Corundum eshold 10700 Pore ratio $26.76 \%$ Fractal dimension 1.848 (6) Gypsum

Figure 9 Fractal dimension of binary CT images.

CT images by the differences in pixel grays. Different pore distributions can produce different density changes that are represented by different gray values in gray CT images. Therefore, the fractal dimensions calculated from gray CT images are larger, which reflects the complex, fine pore structures in basalt, salt rock and coal. Of course, the noise introduced by CT scanning and reconstruction also can affect the results. This is especially the case when the images are flat, but accompanied by much random noise; hence, the calculated results might be a little larger. Hence, a process of noise reduction for CT images should be adopted before making calculations. Results show that the fractal dimen 


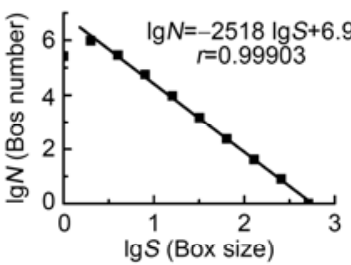

(1) Basalt

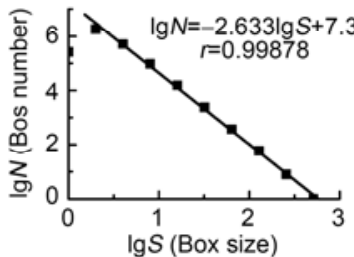

(4) Mud

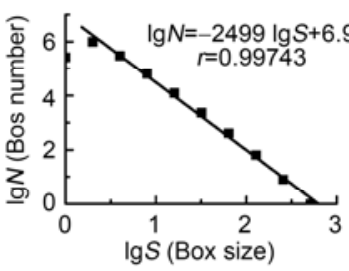

(7) Oil shale

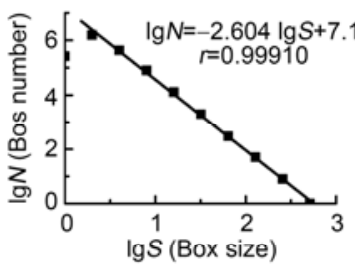

(2) Salt rock

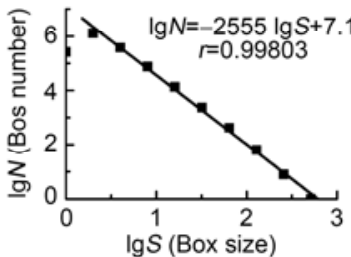

(5) Sandstone

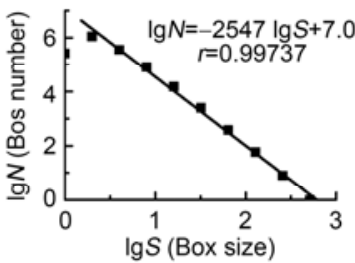

(8) Corundum

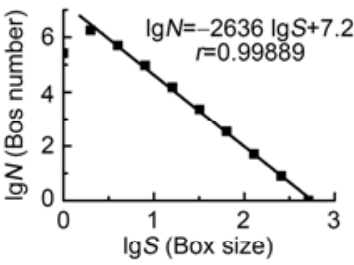

(3) Coal

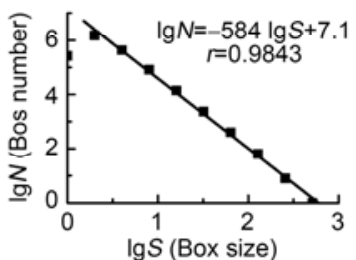

(6) Gypsum

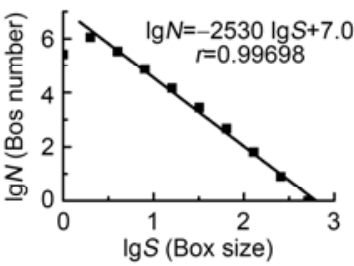

(9) Concrete

Figure 10 Fractal dimensions of gray CT images.

sion of coal is larger than that of salt rock, and the fractal dimension of salt rock is larger than that of basalt. This indicates that the fine pores in coal and salt rock are more complex than in basalt, something that can be verified by other experiments.

The fractal dimensions calculated from gray $\mathrm{CT}$ images of other rock samples are basically consistent with results from the corresponding binary images. The fractal dimension of gypsum and mudstone is greater because of a more complex pore structure across a variety of sizes. The fractal dimension of sandstone, corundum and foam concrete is moderate because of their relatively simple pore structure, which mainly contains some large pores and a few small gaps. The fractal dimension of oil shale is low because of a relatively simple pore structure which mainly contains some larger pores.

It has been demonstrated from the results calculated here that the fractal dimension of coal, salt rock and mudstone are similar and relatively large, although their pore ratios are different. They are sedimentary rocks and thus contain many complex pores with a variety of sizes. However, the minute pores can barely be detected in general porosity measuring tests, and the corresponding binary images fail to reflect this complex micro-pore structure.

The pore ratio and fractal dimensions of different rock samples are listed in Table 2. Figure 11(a) shows the relationship between rock pore ratio and fractal dimensions calculated from the binary CT images. It is evident that the fractal dimension generally increases exponentially with increase in pore ratio. If the pore ratio is small, the changes in pore ratio will lead to a larger variation of fractal dimension. If the pore ratio is large, the changes in pore ratio cause only a small variation in fractal dimension. In Figure 11(b), the relationship between rock pore ratio and fractal dimensions is calculated from gray CT images. Although in general fractal dimensions increase with increases in pore ratios, changes in fractal dimension are more obvious than changes in pore ratio. Thus fractal dimensions can better characterize pore structure in rocks than pore ratios.

\section{Conclusions}

The essential characteristics of images reconstructed by CT scanning of rocks have been discussed in this paper. The pore structures in rocks were extracted from CT images and analyzed. The relationship between pore ratio and fractal dimension of pore structure was evaluated and it was concluded that:
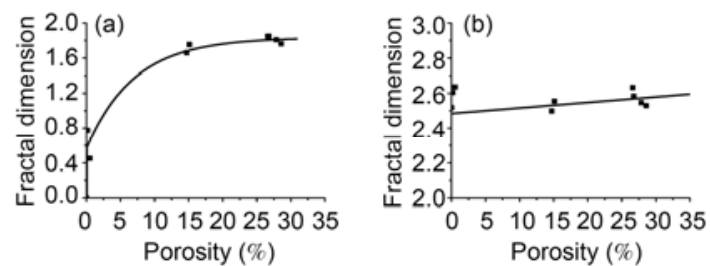

Figure 11 Variety of rock porosity and fractal dimension of rock pores. 
Table 2 Porosity and fractal dimension of various rock samples

\begin{tabular}{ccccc}
\hline Rock class & Section threshold & Pore ratio $(\%)$ & $\begin{array}{c}\text { Fractal dimension of binary } \\
\text { image }\end{array}$ & $\begin{array}{c}\text { Fractal dimension of gray } \\
\text { image }\end{array}$ \\
\hline Basalt & 10000 & 0 & 0 & 2.518 \\
Salt rock & 7000 & 0.17 & 0.775 & 2.604 \\
Coal & 3100 & 0.51 & 0.453 & 2.636 \\
Oil shale & 8500 & 14.71 & 1.661 & 2.499 \\
Concrete & 6000 & 27.85 & 1.718 & 2.530 \\
Sandstone & 10000 & 15.11 & 1.753 & 2.555 \\
Corundum & 6400 & 27.85 & 1.809 & 2.547 \\
Gypsum & 10700 & 26.76 & 1.848 & 2.584 \\
Mud & 10700 & 26.62 & 1.847 & 2.633 \\
\hline
\end{tabular}

(1) The gray value of each pixel in CT images of rocks is a comprehensive reflection of the attenuation coefficient of all the material in the corresponding rock cell. The effect of pores with all kinds of scales in rocks can be represented in gray CT images obtained by scanning with a high resolution industrial real-time CT imaging system.

(2) The contrast in CT images can be effectively enhanced by subsection gray mapping from the 16-bit initial data to the 8-bit gray images. Accordingly all types of pore structures in rocks can be clearly shown.

(3) A segmentation threshold can be determined by inverse analysis based on pore ratios that are measured experimentally, and subsequently binary images of rock pores can be obtained to study the topological structures of rock pores.

(4) The fractal dimension of rock pore structure increases with increase in rock pore ratios. Fractal dimensions might be different even though pore ratios are the same. The more complex the structure of the rocks, the larger the fractal dimension becomes. Therefore, pore structures are better characterized by fractal dimensions. This is especially true for those pore structures with the same pore ratio; whereby the fractal dimension would effectively reflect their differential complexity.

(5) It was experimentally validated that fractal dimension calculations directly from gray CT images of rocks are possible. This is helpful to simplify the calculation process by eliminating binarization that would cause some disturbances and errors. Moreover, this method also reflects the effect of fine micro-pores as much as possible. Therefore, fractal dimensions based on gray CT images complement pore ratios, which can describe the fractal characteristics of pore structures in rocks.

This work was supported by the National Natural Science Foundation of China (10802092, 50974125), the National Basic Research Program of China (2009CB724602, 2010CB226804), the Specialized Research Fund for the Doctoral Program of Higher Education (20070290011) and the Fundamental Research Funds for the Central Universities (2009QM03).

1 Liu P S. Introduction to Cellular Materials (in Chinese). Beijing: Tsinghua University Press, 2004
2 Boer R. Reflections on the development of the theory of porous media. Appl Mech Rev, 2003, 56: R27-42

3 Tsakiroglou C D, Payatakes A C. Characterization of the pore structure of reservoir rocks with the aid of serial sectioning analysis, mercury porosimetry and network simulation. Adv Water Resour, 2000, 23: 773-789

4 Song Y Q, Ryu S G, Sen P N. Determining multiple length scales in rocks. Nature, 2000, 406: 178-181

5 Chen Q, Song Y Q. What is the shape of pores in natural rocks. J Chem Phys, 2002, 116: 8247-8250

6 Radlinski A P, loannidis M A, Hinde A L, et al. Angstrom to millimeter characterization of sedimentary rock microstructure. J Colloid Interf Sci, 2004, 274: 607-612

7 Xie Q, Jiang C X, Ling J M. Meso Mechanics of Rock Experiment and Analysis (in Chinese). Chengdu: Southwest Jiaotong University Press, 1997

8 Raynaud S, Fabre D, Mazerolle F, et al. Analysis of the internal structure of rocks and characterization of mechanical deformation by a non-destructive method: X-ray tomodensitometry. Tectonophysics, 1989, 159: 49-59

9 Buyukozturk O. Imaging of concrete structures. NDT and E Int, 1998, 31: 233-243

$10 \mathrm{Pu}$ Y B. The introduction of applying method for CT in frozen soil experiment research (in Chinese). J Glaciol Geocry, 1993, 15: 196-198

11 Yang G S, Xie D Y, Zhang C Q, et al. CT identification of rock damage properties (in Chinese). Chin J Rock Mech Eng, 1996, 15: 48-54

12 Yang G S, Xie D Y, Zhang C Q, et al. CT detection of damage of coal and rock (in Chinese). Mech Engineer, 1996, 17: 14-16

13 Ge X R, Ren J X, Pu Y B, et al. Real-in time CT test of the rock meso-damage propagation law. Sci China Ser E-Tech Sci, 2001, 44: 328-336

14 Ge X R, Ren J X, Pu Y B, et al. Micro and Meso Experimental Studies on Damage Mechanics for Geotechnical Materials (in Chinese). Beijing: Science Press, 2004

15 Ding W H, Wu Y Q, Pu Y B, et al. History and present situation of $\mathrm{X}$-ray Computerized Tomography (CT) of rocks (in Chinese). Seismolo Geo, 2003, 25: 467-476

16 Cnudde V, Masschaele B, Dierick M, et al. Recent progress in X-ray CT as a geosciences tool. Appl Geochem, 2006, 21:826-832

17 Appoloni C R, Fernandes C P, Rodrigues C R O. X-ray microtomography study of a sandstone reservoir rock. Nucl Instrum Meth A, 2007, 580: 629-632

18 Yang G S, Liu H. Study on the rock damage characteristics based on the technique of CT image processing (in Chinese). J China Coal Soc, 2007, 32: 463-468

19 Yin X T, Dang F N, Ding W H, et al. Quantitative description of crack based on the image measuring technique and CT test (in Chinese). J Expl Mech, 2005, 20: 448-454

$20 \mathrm{Ju} \mathrm{Y}$, Yang Y M, Song Z D, et al. A statistical model for porous structure of rocks. Sci China Ser E-Tech Sci, 2008, 51: 2040-2058

21 Ju Y, Yang Y M, Mao Y Z, et al. Laboratory investigation on mecha- 
nisms of stress wave propagations in porous media. Sci China Ser E-Tech Sci, 2009, 52: 1374-1389

$22 \mathrm{Ju}$ Y, Wang H J, Yang Y M, et al. Numerical simulation of mechanisms of deformation, failure and energy dissipation in porous rock media subjected to wave stresses. Sci China Tech Sci-Tech Sci, 2010, 53: 1098-1113

23 Xie H P. Fractal geometry and its application to rock and soil materials (in Chinese). Chin J Geotech Eng, 1992, 14: 14-24

24 Xie H P. Fractals in Rock Mechanics. Rotterdam: Balkema, 1993

25 Yang P L, Luo Y P, Shi Y C. Fractal feature of soil characterized by weight distribution of grain size. Chinese Sci Bull, 1993, 38: 1896-1899

26 He C Z, Hua M Q. Fractal geometry description of reservoir pore structure (in Chinese). Oil Gas Geology, 1998, 19: 15-23

27 Li T J, Wang Y H, Zhang M Y, et al. Fractal properties of crack in rock and mechanism of rock-burst (in Chinese). Chin J Rock Mech Eng, 2000, 19: 6-10

28 Lian J F, Shen N Q, Zhang J K. Application research on fractal theory in rockmass quality evaluation (in Chinese). Chin J Rock Mech Eng, 2001, 20(Suppl): 1695-1698

29 Xue R, Hu R L, Mao L T. Fractal study on the microstructure variation of soft soils in consolidation process (in Chinese). China Civil Enginee J, 2006, 39: 87-91

30 Fang H W, Chen M H, Chen Z H. Surface pore tension and adsorption characteristics of polluted sediment. Sci China Ser G-Phys Mech Astron, 2008, 51: 1022-1028

31 Zhou H W, Zhang Y H, Li A M, et al. Experimental study on moving boundaries of fluid flow in porous media. Chinese Sci Bull, 2008, 53: 2438-2445

32 Zhang J R, Tao G L, Huang L, et al. Porosity models for determining the pore-size distribution of rocks and soils and their applications. Chinese Sci Bull, 2010, 55: 3960-3970

33 Tao G L, Zhang J R. Two categories of fractal models of rock and soil expressing volume and size-distribution of pores and grains. Chinese Sci Bull, 2009, 54: 4458-4467

34 Feng J, Yu J Y. Determination fractal dimension of soil macropore using computed tomography (in Chinese). J Irrig Drain, 2005, 24: 26-28

35 Zhou S Z, Dang F N, Chen H Q, et al. Fractional characteristics of damage and fracture of concrete under the uniaxial compression CT test (in Chinese). J Hydroelectric Enginee, 2006, 25: 112-117

36 Chen X S, Wu A X, Yang B H, et al. Study on fractal characteristics of pore structure of bulk rock-ore in dump leaching based on CT image (in Chinese). Metal Mine, 2007, 385: 75-77

37 He J, Liu J L, Lv F. Characterizing fractal characteristics of soil pores based on high-resolution digital CT images (in Chinese). Soils, 2008, 40: 662-666

38 Yin X T, Wang S L, Dang F N, et al. Study on fractal characteristics of sandstone damage-fracture under CT test condition (in Chinese). Chin J Rock Mech Eng, 2008, 27(Suppl): 2721-2726

39 Guo D M, Zuo J P, Zhang H, et al. Mechanics of crack propagation in high performance concrete and its fractal dimensions based on ct observation(in Chinese). J Chinese Ceram Soc, 2009, 37: 1607-1612

40 Zhang C Z, Guo Z P, Zhang P, et al. Industrial CT Technology and Principles (in Chinese). Beijing: Science Press, 2009

41 Peng R D, Xie H P, Ju Y. Computation method of fractal dimension for 2-D digital image (in Chinese). J China Univer Min Technolo, 2004, 33: 19-24

42 Yang Y C, Peng R D, Zhou H W. Computation of fractal dimension for digital image in a 3-D space (in Chinese). J China Univer Min Technolo, 2009, 38: 251-258

Open Access This article is distributed under the terms of the Creative Commons Attribution License which permits any use, distribution, and reproduction in any medium, provided the original author(s) and source are credited. 